Original Article

\title{
Anti-inflammatory effects of DA-9601, an extract of Artemisia asiatica, on aceclofenac-induced acute enteritis
}

\author{
Ju Hwan Kim ${ }^{1, \#}$, Chang Yell Shin ${ }^{2, \#}$, Sun Woo Jang ${ }^{2}$, Dong-Seok Kim ${ }^{3}$, Wonae Lee ${ }^{4}$, Hyung-Gun Kim ${ }^{1,5}$, and Hak Rim Kim ${ }^{1, *}$ \\ 'Department of Pharmacology, College of Medicine, Dankook University, Cheonan 31116, ${ }^{2}$ Research Institute of Dong-A ST Co., Ltd., Yongin 17073, \\ ${ }^{3}$ Department of Biochemistry, College of Medicine, Chung-Ang University, Seoul 06974, ${ }^{4}$ Department of Pathology, College of Medicine, Dankook University, \\ Cheonan 31116, ${ }^{5}$ NeuroVis Inc., Cheonan 31035, Korea
}

\author{
ARTICLE INFO \\ Received February 26, 2021 \\ Revised March 16, 2021 \\ Accepted May 6, 2021 \\ *Correspondence \\ Hak Rim Kim \\ E-mail: hrkim@dankook.ac.kr
}

\section{Key Words}

DA-9601

Inflammation

NSAIDs

Small intestine

T cell

"These authors contributed equally to this work.
ABSTRACT DA-9601 is an extract obtained from Artemisia asiatica, which has been reported to have anti-inflammatory effects on gastrointestinal lesions; however, its possible anti-inflammatory effects on the small intestine have not been studied yet. Therefore, in this study, we investigated the protective effects of DA-9601 against the ACF-induced small intestinal inflammation. Inflammation of the small intestine was confirmed by histological studies and the changes in the $\mathrm{CD}^{+} \mathrm{T}$ cell fraction induced by the inflammation-related cytokines, and the inflammatory reactions were analyzed. Multifocal discrete small necrotic ulcers with intervening normal mucosa were frequently observed after treatment with ACF. The expression of IL-6, IL17 , and TNF- $\alpha$ genes was increased in the ACF group; however, it was found to have been significantly decreased in the DA-9601 treated group. In addition, DA-9601 significantly decreased the levels of proinflammatory mediators such as IL-1 $\beta$, GMCSF, IFN- $\gamma$, and TNF- $\alpha$; the anti-inflammatory cytokine IL-10, on the other hand, was observed to have increased. It is known that inflammatory mediators related to T cell imbalance and dysfunction continuously activate the inflammatory response, causing chronic tissue damage. The fractions of IFN- $\gamma^{+}$Th1 cells, IL-4 ${ }^{+}$Th2 cells, IL- $9^{+}$Th 9 cells, IL-17 $7^{+}$Th17 cells, and Foxp $3^{+}$Treg cells were significantly decreased upon DA9601 treatment. These data suggest that the inflammatory response induced by ACF is reduced by DA-9601 via lowering of the expression of genes encoding the inflammatory cytokines and the concentration of inflammatory mediators. Furthermore, DA-9601 inhibited the acute inflammatory response mediated by T cells, resulting in an improvement in ACF-induced enteritis.

\section{INTRODUCTION}

The inflammatory bowel diseases such as Crohn's disease or ulcerative colitis have been gradually increasing in recent years; however, the etiology and pathogenesis of inflammatory bowel disease remain unclear [1]. It is known that a person with genetic vulnerability, when exposed to environmental changes or triggers, develops inflammation and an immune response to the intestinal mucosa, resulting in chronic tissue damage [2]. Many in- flammatory mediators are involved in the inflammatory response to inflammatory bowel disease. In particular, mechanisms related to the production of leukotrienes as inflammatory mediators are expected to be therapeutic targets [3].

The use of nonsteroidal anti-inflammatory drugs (NSAIDs) and the onset of inflammatory bowel disease are known to be related to each other. The development of ulcerative enteritis and Crohn's disease is stimulated by the onset and/or exacerbation of inflammatory bowel disease caused by using NSAIDs [4]. Clini- (i) (\$)

This is an Open Access article distributed under the terms of the Creative Commons Attribution Non-Commercial License, which permits unrestricted non-commercial use, distribution, and reproduction in any medium, provided the original work is properly cited. Copyright $\odot$ Korean J Physiol Pharmacol, pISSN 1226-4512, elSSN 2093-3827
Author contributions: C.Y.S., S.W.J., H.G.K., and H.R.K. conceived and designed the experiment. J.H.K., W.L., D.S.K., and H.R.K. performed experiments and analyzed the data. C.Y.S. and H.R.K. wrote the manuscript with input from all authors. All authors have read and agreed to the published version of the manuscript. 
cally, the prevalence of inflammatory bowel disease in patients have been found to be related to the use of NSAIDs and hence are recommended to limit the use of NSAIDs [5].

Proton-pump inhibitors (PPIs) strongly inhibit gastric acid secretion in the stomach and have been widely used for the treatment of upper gastrointestinal ulcerative lesions. However, some reports have suggested that PPIs cause an imbalance in the small intestinal bacterial flora, further exacerbating the NSAIDinduced small intestinal injury [6-8]. The association between the PPI treatment and prognosis of enteritis has not been clearly revealed yet.

Aceclofenac (ACF), one of the commonly used NSAIDs, has been used to relieve the pain caused by rheumatoid arthritis, ankylosing spondylitis, osteoarthritis (degenerative arthritis), periarthritis of the scapular humerus, and toothache [9]. However, ACF may increase the risk of serious gastrointestinal adverse events including gastric or intestinal bleeding, ulcers, and perforation. These adverse reactions have emerged as a factor that limits the prescription for patients, such as those suffering from rheumatoid arthritis, who require long-term administration of ACF [9].

DA-9601 is an ethanol extract of Artemisia asiatica and is currently used as a clinical medicine for the treatment of gastritis [10]. Interestingly, it has been reported to have anti-oxidative and antiinflammatory effects on experimentally induced gastrointestinal, hepatic, and pancreatic lesions $[11,12]$. However, its possible antiinflammatory effects on the small intestine have not been studied yet. Therefore, in this study, we investigated the protective effects of DA-9601 against the ACF-induced small intestinal inflammation.

\section{METHODS}

\section{Reagents}

All reagents were provided from Dong-A ST (Yongin, Korea). DA-9601, the standardized ethanol extract of Artemisia asiatica, was supplied by Dong-A Pharmaceutical Co. Ltd. (Yongin, Korea). The percentage of eupatilin, a pharmacologically active ingredient of Artemisia asiatica, was found to be $2.25 \%$ (w/w) as determined by HPLC. DA-9601 was dissolved in $5 \mathrm{ml} \mathrm{3 \%}$ hydroxypropyl methylcellulose (HPMC) (Sigma-Aldrich, St. Louis, MO, USA) solution.

\section{Animals}

Six-week-old male Sprague Dawley (SD) rats were obtained from Daehan Bio Link (Eumseong, Korea); those with body weights of approximately 200-250 g were used for the experiments. Animals were acclimated for a week and maintained at an ambient temperature of $23 \pm 3^{\circ} \mathrm{C}$ with 12 -h light/dark cycles (lights on 07:00-19:00). Food pellets (Daehan Bio Link) and wa- ter were provided ad libitum. After a 1-week adaptation period, rats were randomly assigned to five groups of 8-10 rats each. All animal procedures adhered to the National Institutes of Health Guidelines for Animal Research and were approved by the Dankook University Institutional Animal Care and Use Committee (IACUC; DKU-15-001), which adheres to the guidelines issued by the Institution of Laboratory Animal Resources.

\section{Experimental design}

Male SD rats weighing around 200-250 g were divided into five groups of 8-10 rats each. The first group was a control group treated with 3\% HPMC for 4 days. The second group was pretreated with 3\% HPMC, and ACF was then orally administered (50 mg/kg body weight) once a day for 2 days. The third group was pretreated with DA-9601 (100 mg/kg body weight) for 2 days and then co-treated with ACF (50 mg/kg) + DA-9601 (100 mg/ $\mathrm{kg}$ ) for 2 days. The fourth group was pretreated with esomeprazole (ESO) (10 mg/kg body weight) for 2 days and then co-treated with ACF $(50 \mathrm{mg} / \mathrm{kg})+\mathrm{ESO}(10 \mathrm{mg} / \mathrm{kg})$ for 2 days. The last group was pretreated with DA-9601 (100 mg/kg) + ESO $(10 \mathrm{mg} / \mathrm{kg})$ for 2 days and then co-treated with ACF $(50 \mathrm{mg} / \mathrm{kg})+$ DA-9601 (100 $\mathrm{mg} / \mathrm{kg})+\mathrm{ESO}(10 \mathrm{mg} / \mathrm{kg})$ for 2 days. Two-day consecutive dosing of ACF was selected from a preliminary study that measured small intestinal inflammatory lesions; in this study, treatment with $50 \mathrm{mg} / \mathrm{kg}$ of ACF for two consecutive days was observed to cause detectable ulcers in the small intestine. One-day dosing did not cause apparent inflammatory lesions in the small intestine. ACF dose of 100-200 mg/kg was observed to cause severe small intestinal inflammatory lesions and hence was not deemed appropriate for this study. Post $24 \mathrm{~h}$ of the last drug administration, the rats were euthanized by ether anesthesia. The small intestine, from the gastric duodenum to the large intestine, was quickly removed by incising the abdomen. The excised small intestine was washed with sterilized physiological saline to remove feces. Samples collected from each experimental group for the analysis of inflammatory mediators were stored at $-80^{\circ} \mathrm{C}$ till further investigation.

\section{Visual observation of inflammation and histological analysis}

The freshly excised small intestine was thoroughly washed with sterilized physiological saline to remove feces, and $1 \%$ paraformaldehyde was immediately added for fixation, for $30 \mathrm{~min}$. Fixed tissues were spread out and visually observed. Inflammation lesions of the small intestine were mainly found in the lower intestine; hence, the lower part of the intestine was used for subsequent experiments. Data on the visual observation were collected using photography, and a quantitative analysis of the inflammation was performed using the following observation score criteria (Tables 1, 2). Small intestine tissue samples $(1 \mathrm{~cm} \times 1 \mathrm{~cm})$ from experimental 
Table 1. Observation score criteria of inflammation

\begin{tabular}{cl}
\hline Score & \multicolumn{1}{c}{ Tissue condition } \\
\hline 0 & No lesions \\
1 & Punctiform lesions $(<1 \mathrm{~mm})$ \\
2 & Five or more punctiform lesions \\
3 & One to five small ulcers $(1-2 \mathrm{~mm})$ \\
4 & More than five small ulcers or one large ulcer \\
5 & More than one large ulcer (greater than $4 \mathrm{~mm})$ \\
\hline
\end{tabular}

animals were fixed in $4 \%$ neutral buffered formaldehyde and processed by routine techniques prior to embedding in the paraffin blocks in the pathology lab at Dankook University Hospital. Briefly, a thick section $(4 \mu \mathrm{m})$ was mounted on a glass slide and stained with hematoxylin and eosin. The sections were examined under a light microscope (CK-40; Olympus, Tokyo, Japan) by a pathologist who was blinded to the treatment.

\section{Gene expression profile for inflammatory mediator}

Total RNA was isolated from the collected samples by using TRIzol reagent (Thermo Fisher Scientific, Waltham, MA, USA). RNA was reverse transcribed into CDNA by using MMLV reverse transcriptase (Bioneer, Daejeon, Korea) and oligo-d(T) primer. Quantitative real time PCR (qRT-PCR) was performed using a Rotor Gene SYBR Green Supermix Kit (Qiagen, Hilden, Germany), and the fluorescence was measured using a Rotorgene PCR Cycler (Qiagen). Glyceraldehyde 3-phosphate dehydrogenase (GAPDH) was used as a reference gene. The primers used for qRT-PCR were synthesized by Cosmo Genetech (Seoul, Korea). The sequences of the forward and reverse primers used in the qRT-PCR and Semi-quantitative RT-PCR (sqRT-PCR) are as follows: IL-6 (275 bp), forward 5'-GGAGTTCCGTTTCTACCTGG-3' and reverse 5'-GCCGAGTAGACCTCATAGTG-3'; TNF- $\alpha$ (316 bp), forward 5'-CCACGTCGTAGCAAACCACCAAG-3' and reverse 5'-CAGGTACATGGGCTCATACC-3'; IL17 (178 bp), forward 5'-ACAGTGAAGGCAGCGGTACT-3' and reverse 5'-GCTCAGAGTCCAGGGTGAAG-3'; GAPDH (134 bp), forward $5^{\prime}$-AACGGCACAGTCAAGGCTGA-3' and reverse 5'-ACGCCAGTAGACTCCACGACAT-3'. The relative expression levels of $I L-6, I L-17$, and TNF- $\alpha$ were measured as an index of gene expression related to inflammation. Three biologically independent experiments were performed, and each PCR reaction was repeated in triplicates. The relative level of a specific mRNA was calculated by normalizing its expression to that of GAPDH by using the $2^{-\triangle \Delta C t}$ method. The sqRT-PCR reactions were performed using PCR PreMix (Bioneer). The PCR product of each gene was analyzed using $1.5 \%$ agarose gel electrophoresis, the bands were stained using Syto 60 (Li-COR Biosciences, Lincoln, $\mathrm{NB}, \mathrm{USA}$ ), and the stained gel was visualized using the Odyssey infrared imaging system (Li-COR Biosciences).
Table 2. Observation score of inflammation

\begin{tabular}{lc}
\hline \multicolumn{1}{c}{ Group } & Inflammation score \\
\hline Normal & $0.3 \pm 0.48$ \\
ACF & $4.6 \pm 0.67$ \\
ACF + DA-9601 & $3.7 \pm 1.10^{*}$ \\
ACF + ESO & $4.5 \pm 0.93$ \\
ACF + DA-9601 + ESO & $3.8 \pm 0.94^{*}$ \\
\hline
\end{tabular}

Data are represented as the mean \pm SEM. ACF, aceclofenac; ESO, esomeprazole. ${ }^{*} p<0.05$ vs. ACF.

\section{Analysis of inflammatory mediators in small intestine}

Samples were prepared according to the manufacturer's instructions, and the chemokines or cytokines were quantified using an ELISA kit. Concentrations of IL-6, IL-1 $\beta$, IL-10, GMCSF, IFN- $\gamma$, and TNF- $\alpha$ in the small intestines of the drug-treated rats $(50 \mathrm{mg} / \mathrm{kg}$ of $\mathrm{ACE}$ ) were determined using an ELISA kit (Ab frontier, South Korea) following the manufacturer's protocol. The concentration of whole homogeneous lysates obtained from each sample was normalized and the lysates were diluted within the measurable range. Optical density was measured at $450 \mathrm{~nm}$ (A450) using a microplate reader (Thermo Scientific Multiskan GO, Microplate Spectrophotometer; Thermo Fisher Scientific). Briefly, small intestine tissue $(100 \mathrm{mg})$ was washed with $1 \mathrm{X}$ PBS, and then $1 \mathrm{ml}$ of $1 \mathrm{X}$ PBS was added to completely homogenize the isolated small intestine by using a tissue disperser homogenizer. Homogenates were centrifuged at $5,000 \mathrm{~g}$ for $5 \mathrm{~min}$ at $2-8^{\circ} \mathrm{C}$. The supernatant was used to measure the enzyme activity. To normalize the protein levels in all supernatant samples, the protein concentration was quantified in each sample by using the Lowry protein assay kit (Bio-Rad, Hercules, CA, USA).

\section{Flow cytometric analysis}

The small intestine samples were washed 3 times with 1X PBS at $4^{\circ} \mathrm{C}$, and then the intestinal epithelium was separated with cell separation solution at $37^{\circ} \mathrm{C}$. The cell separation solution was composed of $10 \%$ fetal bovine serum, $20 \mathrm{mM}$ Hank's balanced salt solution, $100 \mathrm{U} / \mathrm{ml}$ penicillin, $100 \mu \mathrm{g} / \mathrm{ml}$ streptomycin, $1 \mathrm{mM}$ sodium pyruvate, $10 \mathrm{mM}$ EDTA, and $10 \mu \mathrm{g} / \mathrm{ml}$ polymyxin B. After washing 5 times with 1X PBS, RPMI 1640 (Sigma-Aldrich) containing $400 \mathrm{U} / \mathrm{ml}$ collagenase D (Roche, Mannheim, Germany) and $10 \mu \mathrm{g} / \mathrm{ml}$ DNase I (Roche) was added along with $10 \% \mathrm{FBS}$, and the samples were incubated at $37^{\circ} \mathrm{C}$ for $90 \mathrm{~min}$. Subsequently, $10 \mathrm{mM}$ EDTA was added, and the samples were subjected to flow cytometry. The cells in the small intestine were analyzed by flow cytometry on the FACSCalibur system using the Cell Quest software (Becton Dickinson, Franklin Lakes, NJ, USA). The antibodies used were phycoerythrin (PE)-binding anti-mouse CD4 (GK1.5, Rat IgG2b, א), peridinin-chlorophyll protein-binding anti-mouse CD4 (GK1.5), fluorescein isothiocyanate-binding anti- 
mouse IFN- $\gamma$ (XMG1.2, Rat IgG1, $\kappa)$, PE-binding anti-mouse IL4, PE-binding anti-mouse IL-9 (RM9A4, Rat IgG1, $\kappa)$, PE-binding anti-mouse IL-17A (TC11-18H10.1, Rat IgG1, $\kappa)$, and Alexa Fluor 488 binding anti-mouse Foxp3 (150D, Mouse IgG1, א) (Biolegend, San Diego, CA, USA). Fifty thousand single cells were analyzed per experiment.

\section{Statistical analysis}

All data are presented as the mean \pm SEM. All statistical analyses were performed using GraphPad Prism (GraphPad Software, La Jolla, CA, USA). Student's t-test was used for comparing the data between two groups, whereas one-way analysis of variance
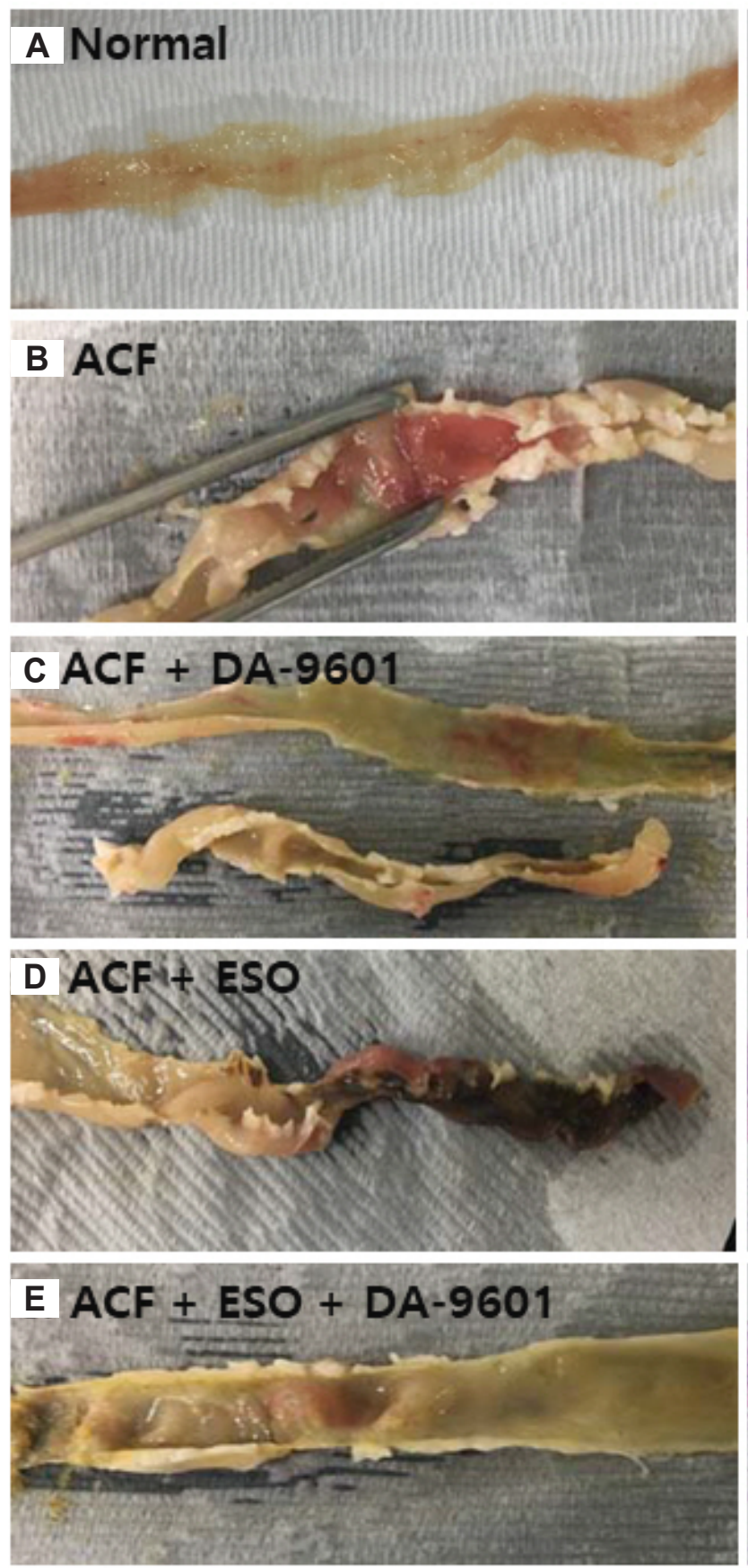

\section{X}
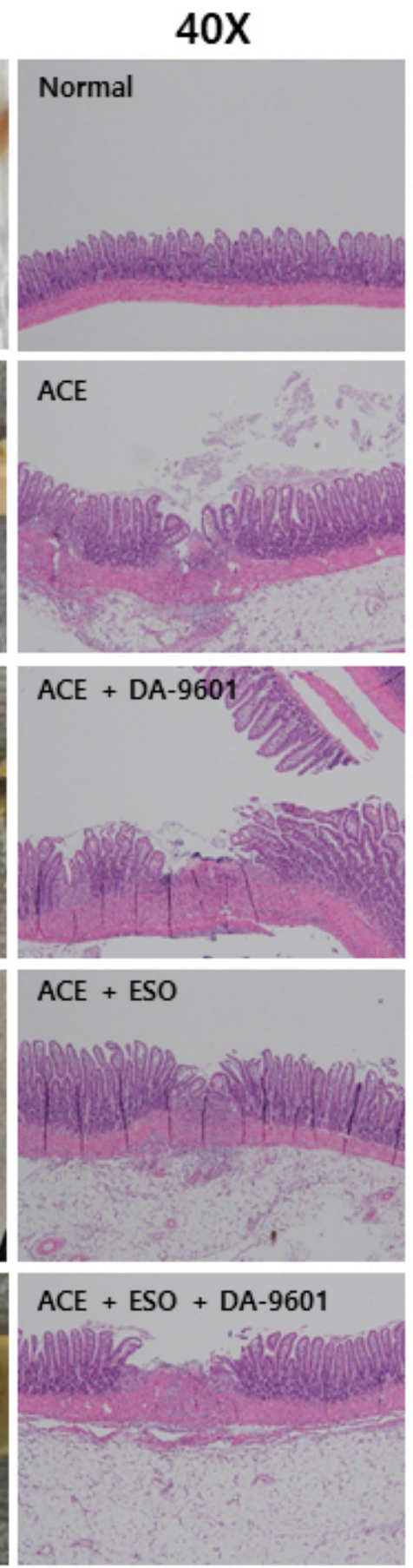

$100 x$
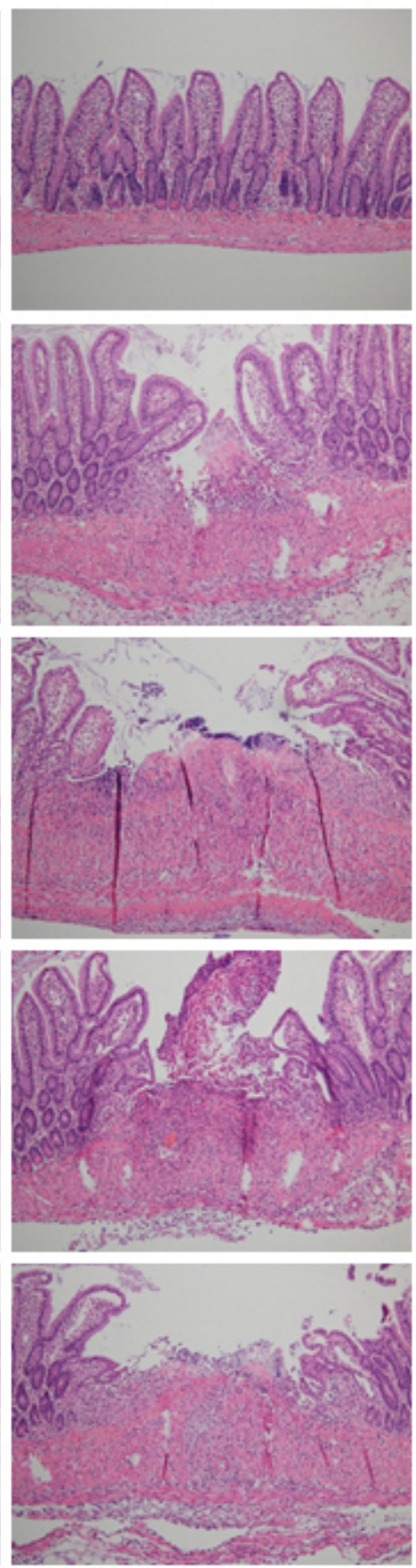

Fig. 1. Representative images depicting histological appearance of small intestine tissues after ACF-induced enteropathy. (A) Small intestine section from the normal control group. Small intestine section from (B) ACF treated group (C) ACF + DA-9601 treated group, (D) ACF + ESO treated group, and (E) ACF + ESO + DA-9601 treated group. Multifocal discrete small necrotic ulcers with intervening normal mucosa were observed. Frequent necrotic ulcers through small intestine were also observed, and the degrees of necrotic damage were considered very severe with accompanying intestinal micro perforations with peritonitis ( $\mathrm{H} \& \mathrm{E}$, magnification: $\times 40$ and $\times 100)$. ACF, aceclofenac; ESO, esomeprazole. 
(ANOVA) was used for comparisons of data from more than three groups. The significance for all comparisons of interest was assessed, and $\mathrm{p}<0.05$ was considered statistically significant.

\section{RESULTS}

\section{Histological evaluation of the severity of inflammation of small intestine}

The severity of inflammation was evaluated using the observation score of inflammation (Tables 1,2), and the experimental observations were blinded for maintaining the objectivity of the data. In the control group, no significant ulcers, necrosis, abscesses etc. were observed in the small intestine (Fig. 1A). When ACF $(50 \mathrm{mg} / \mathrm{kg}$ ) was administered for 2 days, many extensive ulcer sites were observed, and the inflammation score was counted as 5, which represents the most severe stage (Fig. 1B). Lesions were observed in the form of multifocal discrete small necrotic ulcers with intervening normal mucosa. The activities of the experimental animals slowed after ACF administration; however, no death was reported during the study. When DA-9601(100 mg/ $\mathrm{kg}$ ) was administered concurrently with ACF, significantly fewer ulcer sites than when ACF was administered alone were observed in the small intestine; the inflammation score tended towards 3-4 (Fig. 1C, E). However, when ESO $(10 \mathrm{mg} / \mathrm{kg})$ was administered concurrently with ACF, no significant change in the inflammation score as compared to that with the administration of ACF alone was observed (Fig. 1D).

\section{The effects of DA-9601 or ESO on gene expression of inflammatory mediators}

The gene expression of inflammatory mediators such as IL6 , IL-17, and TNF- $\alpha$ in ACF-induced enteritis was analyzed. The expression of IL- 6 was significantly increased by ACF administration compared to that in the controls. However, the expression of IL- 6 was significantly decreased in the ACF + DA-9601 treated group as compared to that in the ACF treated group. Interestingly, the expression of $I L-6$ was found to have significantly increased in the group treated with ACF + ESO (Fig. 2A). The expression of $I L-17$ in the ACF treated group was significantly increased as compared to that in the control group; however, the expression of IL-17 was found to have significantly decreased in the ACF + DA9601 treated group compared to that in the ACF treated group. The expression of $I L-17$ was the highest in the group treated with ACF + ESO (Fig. 2B). The expression of TNF- $\alpha$ in the ACF treated group was significantly increased compared to that in the control group. The expression of TNF- $\alpha$ was also significantly increased in the group treated with ACF + ESO as compared to the ACF treated group. However, the expression of TNF- $\alpha$ was found to have significantly decreased in the ACF + DA-9601 treated
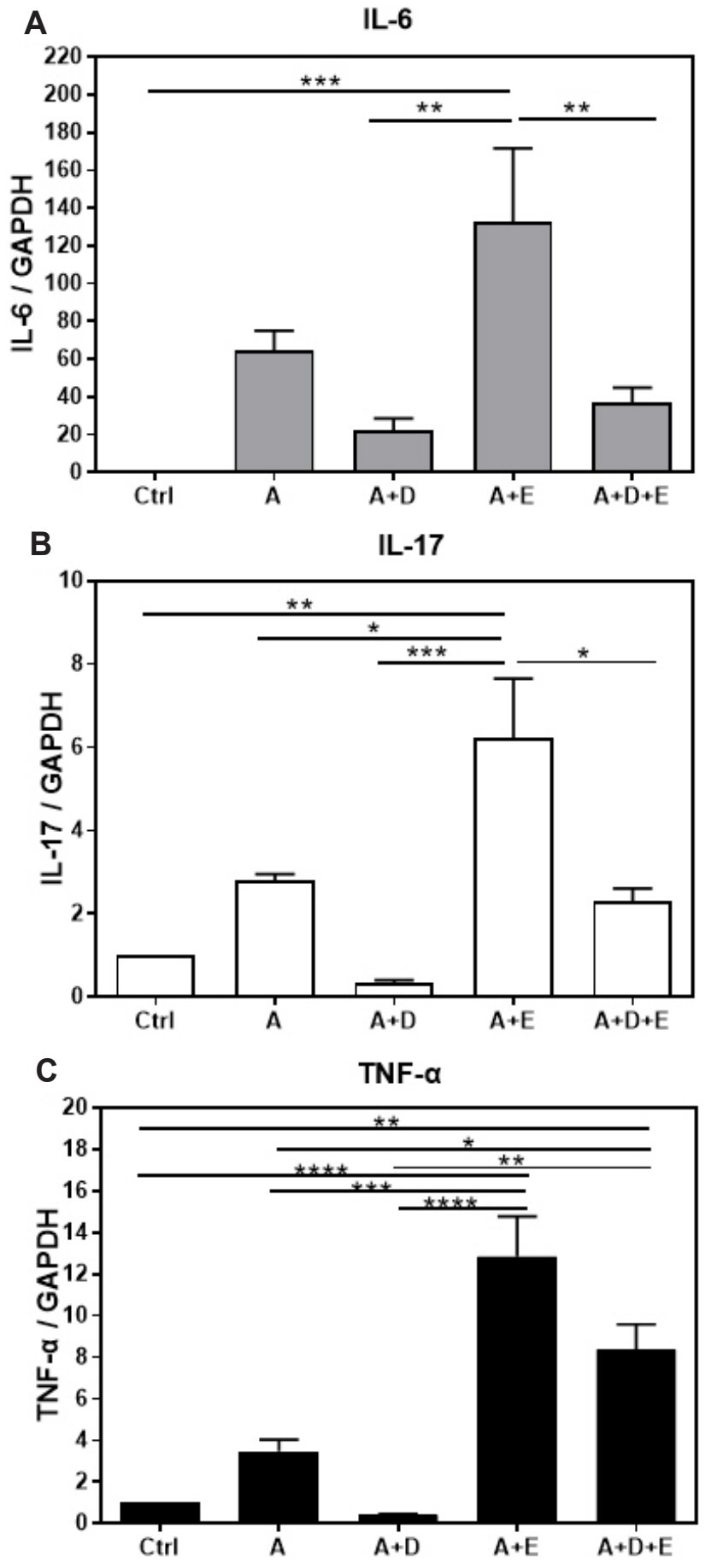

Fig. 2. Expression levels of IL-6, IL-17, and TNF- $\alpha$ mRNA in small intestine after ACF-induced enteropathy. Expression of $I L-6$ (A), IL-17 (B), and TNF- $\alpha(C)$ was quantified using qRT-PCR. The relative mRNA levels of $I L-6, I L-17$, and $T N F-\alpha$ were calculated by normalizing them to the expression level of GAPDH by using the $2^{-\Delta \Delta \mathrm{t}}$ method. The expression values obtained from the drug-treated small intestine were normalized to those of the control. Data are represented as the mean \pm SEM. A, aceclofenac (ACF); D, DA-9601; E esomeprazole. ${ }^{*} p<0.05,{ }^{* *} p<0.01$, *** $p<0.001$, and ${ }^{* * * *} p<0.0001$. 
groups as compared to that in the ACF treated group (Fig. 2C). When ACF + ESO + DA-9601 was administered concurrently, a significant decrease in the expression of genes encoding for inflammatory mediators was observed as compared to the gene expression levels observed in the ACF + ESO treated group; nonetheless, the expression of these genes did not decrease as much as in the ACF + DA-9601 treated group. In particular, the expression of the gene encoding for TNF- $\alpha$ was significantly decreased in the group treated with ACF + ESO + DA-9601 as compared to that in the group treated with ACF + ESO; the expression level was still significantly higher than that in the group treated with ACF alone.

\section{The effects of DA-9601 or ESO on the production of inflammatory mediators}

Unlike gene expression, the concentration of IL-6 in the small intestine was not significantly changed by ACF treatment. In addition, no change was induced by the ACF + DA-9601 treatment. However, the IL- 6 concentration was significantly decreased in the ACF + ESO treated group; the level was restored to the control level by the ACF + ESO + DA-9601 combination treatment (Fig. $3 A)$. The concentration of IL-10, an anti-inflammatory cytokine known as human cytokine synthesis inhibitory factor, was significantly decreased by ESO in ACF-induced enteritis but was significantly increased in the ACF + DA-9601 and ACF + ESO + DA-9601 treatment groups (Fig. 3B). In the ACF-induced enteritis, the concentrations of IL-1 $\beta$ and GM-CSF were significantly increased compared to control; however, when DA-9601 or ESO was concurrently applied with ACF, the concentrations of IL-1 $\beta$ and GM-CSF were significantly decreased. On the other hand, the concentrations of IL-1 $\beta$ and GM-CSF were found to have been significantly increased in the ACF + ESO + DA-9601 treated group (Fig. 3C, D). The concentrations of IFNs and TNF- $\alpha$, which are crucial inflammatory cytokines, were found to have significantly increased in ACF-induced enteritis. However, both cytokines were significantly decreased in the ACF + DA-9601 and $\mathrm{ACF}+\mathrm{ESO}$ treated groups. In addition, the concentrations of interferons (IFNs) and TNF- $\alpha$ were found to have significantly decreased by ACF + ESO + DA-9601 treatment (Fig. 3E, F). IFNs and TNF- $\alpha$ have been suggested as the crucial molecules in the inflammatory processes and are thought to influence cellular, tis-
A

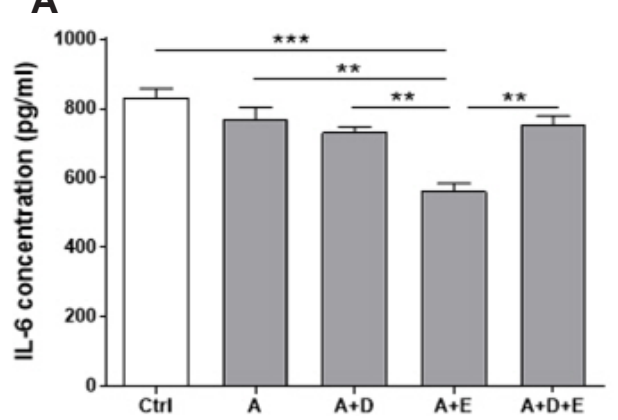

C

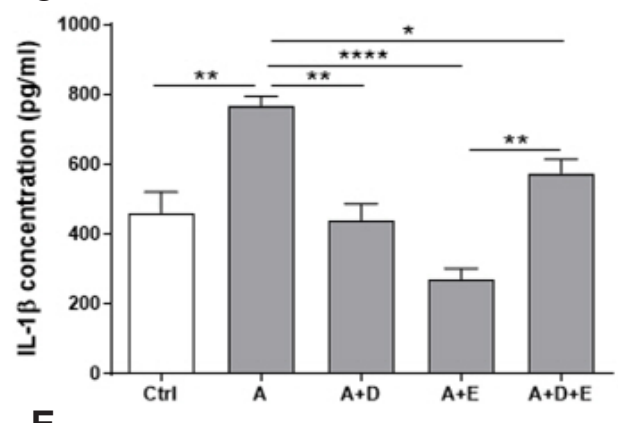

E

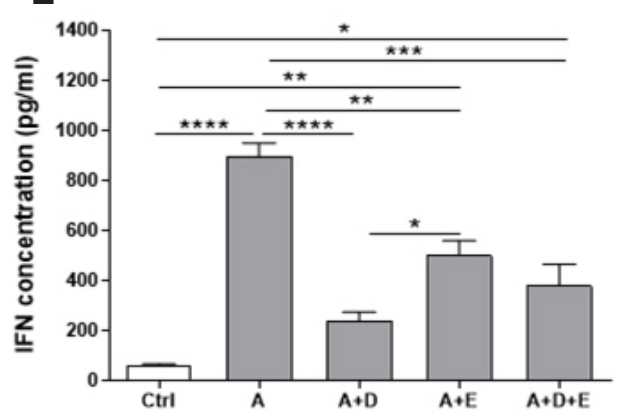

B

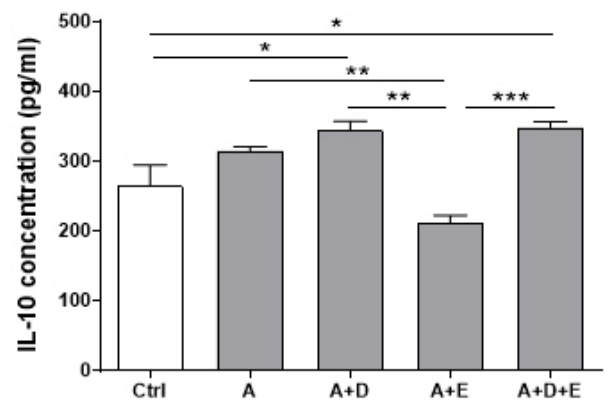

D

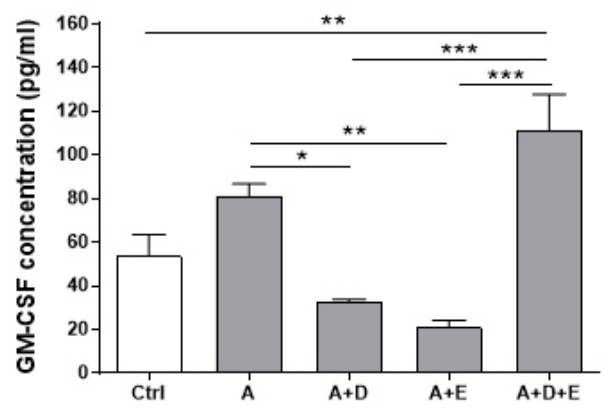

F

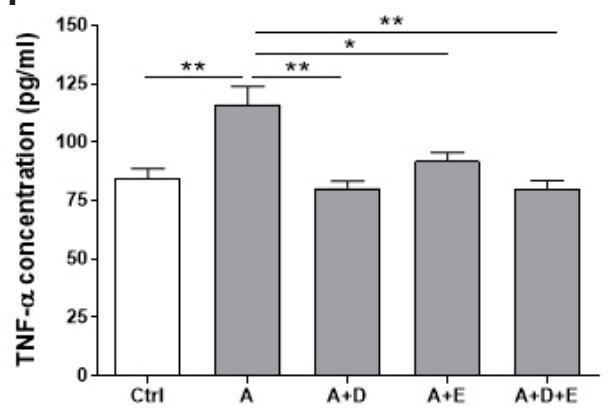

Fig. 3. Concentrations of IL-6, IL-1 $\beta$, IL10 , GM-CSF, IFN- $\gamma$, and TNF- $\alpha$ in small intestine after ACF-induced enteropathy. Quantification of (A) IL-6, (B) IL-10, (C) IL-1 $\beta$, (D) GM-CSF, (E) IFN- $\gamma$, and (F) TNF- $\alpha$ in the small intestine by using ELISA. The concentration of whole lysates homogenized from each sample was normalized, and the lysates were diluted within the measurable range. Statistical significance was evaluated using one-way ANOVA. Data are represented as mean \pm SEM. A, aceclofenac (ACF); D, DA-9601; E, esomeprazole. ${ }^{*} p<0.05,{ }^{* *} p<0.01,{ }^{* * *} p$ $<0.001,{ }^{* * * *} \mathrm{p}<0.0001$. 
sue, and global physiological functions.

\section{Characteristics of $\mathrm{CD} 4^{+} \mathrm{T}$ cell of small intestine in ACF- induced enteritis}

In inflammatory bowel disease, inflammatory mediators related to $\mathrm{T}$ cell imbalance and dysfunction are known to continuously activate the inflammatory response, thus causing chronic tissue damage [2]. The characteristics of $\mathrm{CD} 4^{+} \mathrm{T}$ cells in the small intestine were analyzed using flow cytometry (Fig. 4). Fig. 4A shows 'dot plot' analysis displaying target cell populations and Fig. 4B demonstrates relative percentage of target cell populations for easy understanding of the dot plots. IFN $-\gamma^{+} \mathrm{CD} 4^{+}$cells were significantly increased, compared to the control, in ACF-induced enteritis. There was no significant change in the ACF + DA-9601 treated group, but the fraction was found to have increased by ESO treatment. However, IFN- $\gamma^{+} \mathrm{CD}^{+}{ }^{+}$cell fraction was observed to have decreased by the concurrent treatment of ACF + ESO + DA-9601 (Fig. 4B-a). IL- $4^{+} \mathrm{CD}^{+}$cells also increased in ACFinduced enteritis. These cells were reduced in the ACF + DA-

A
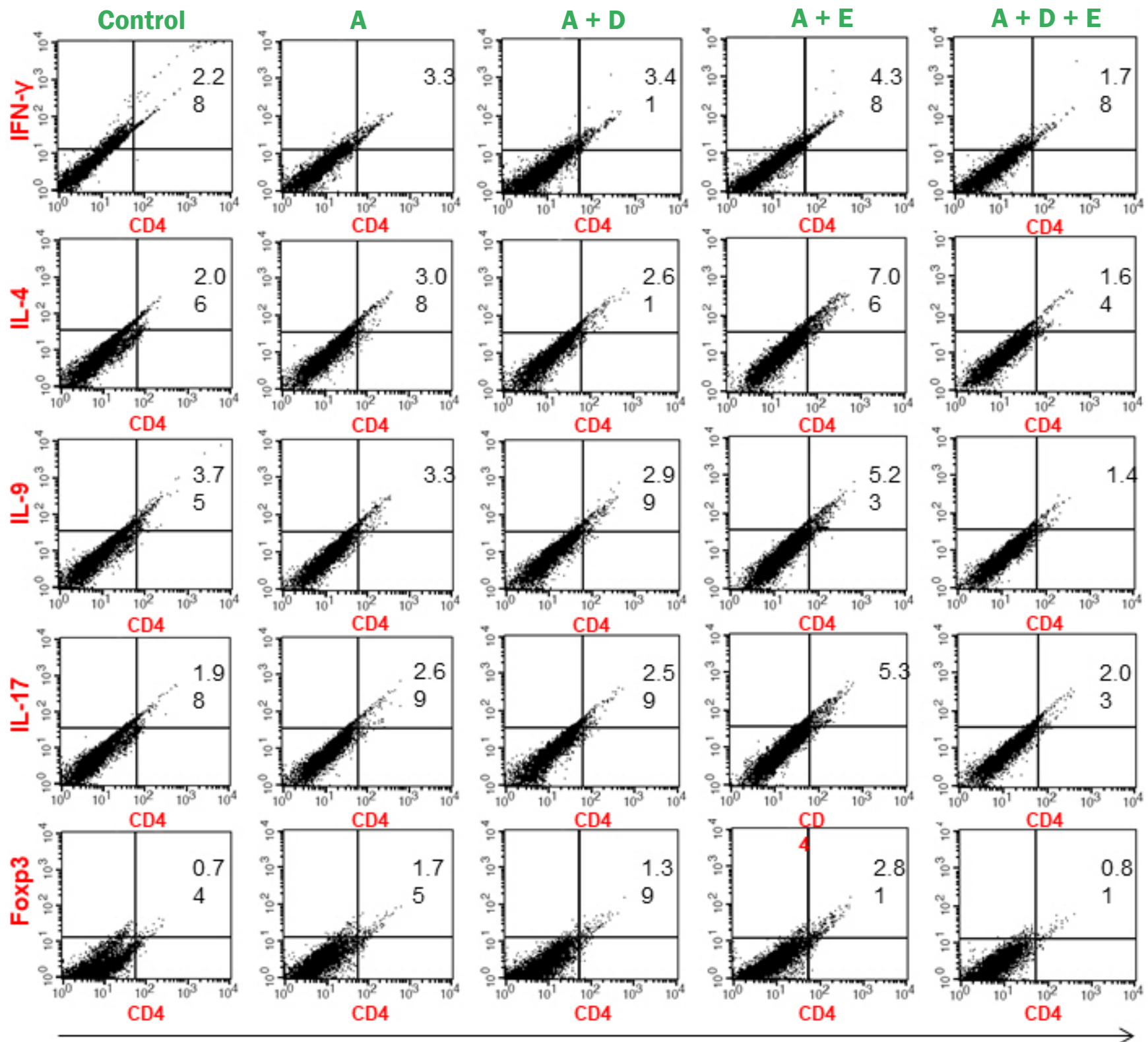

CD4+

Fig. 4. Flow cytometric characteristics of $C D 4^{+} T$ cells in small intestine after ACF- induced enteropathy. (A) Numbers inside quadrant indicate the percentage of the cell population. Only $30 \%$ of the cell populations were presented in the flow cytometric characteristics graph. (B) The proportion of $\mathrm{CD}^{+} \mathrm{T}$ cells from lamina propria was determined according to intracellular levels of cytokines for (a) interferon (IFN)- $\gamma^{+}$Th1 cell, (b) interleukin (IL)- $4^{+}$Th2 cells, (c) IL-9+ Th9 cells, (d) IL-17 Th17 cells, and (e) Foxp3 ${ }^{+}$Treg cells. A, aceclofenac (ACF); D, DA-9601; E, esomeprazole. 
B
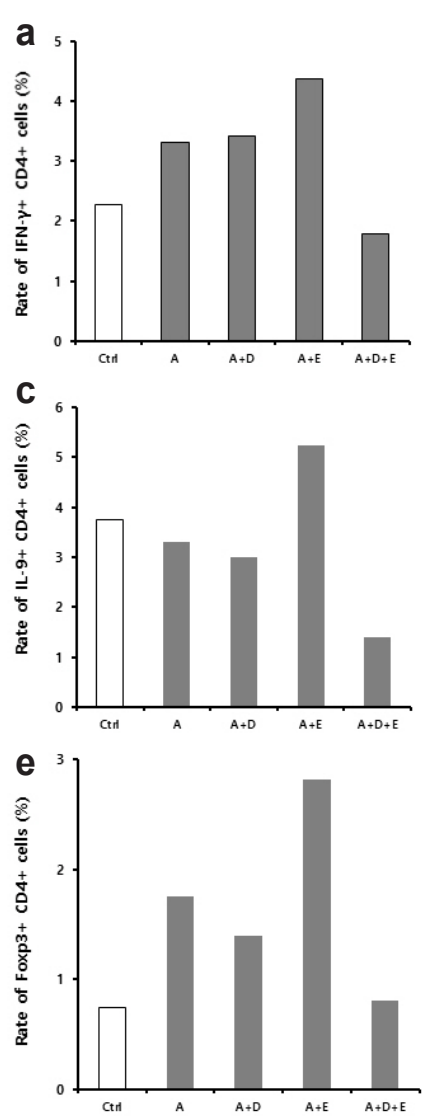

Fig. 4. Continued.

9601 treated group, but significantly increased in the ACF + ESO treated group. Similar to IFN- $\gamma^{+} \mathrm{CD} 4^{+} \mathrm{T}$ cells, $\mathrm{IL}-4^{+} \mathrm{CD} 4^{+}$cells decreased by the concurrent treatment with ACF + ESO + DA9601 (Fig. 4B-b). IL-9 $9^{+} \mathrm{CD}^{+}$cells showed no significant change compared to the control in ACF-induced enteritis. These cells were found to have decreased in the ACF + DA-9601 and ACF + ESO + DA-9601 treated groups but increased upon treatment with ACF + ESO (Fig. 4B-c). IL-17 $\mathrm{CD}^{+}$cells were increased in ACF-induced enteritis. No significant change was caused by ACF + DA-9601 treatment, but these cells were increased upon ACF + ESO treatment. Compared to the ACF + ESO treated group, $\mathrm{IL}-17^{+} \mathrm{CD} 4^{+}$cells further decreased in the ACF + ESO + DA9601 treated group (Fig. 4B-d). Regulatory T cells suppress the immune response; therefore, in animal models, regulatory $\mathrm{T}$ cells are known to be effective in treating enteritis. Foxp $3^{+} \mathrm{CD} 4^{+}$cells were found to have increased in ACF-induced enteritis. It was decreased in the ACF + DA-9601 group but increased in the ACF + ESO group. Compared to the ACF + ESO group, it was decreased in the ACF + ESO + DA-9601 group, and the level of Foxp $3^{+} \mathrm{CD} 4^{+}$ cells returned to normal control levels (Fig. 4B-e).

\section{DISCUSSION}

NSAIDs are widely used for the treatment of numerous inflammatory diseases, including osteoarthritis, despite their adverse effects on the gastrointestinal tract [13]. The damage caused by these drugs in the stomach and duodenum can be reduced by co-administration of inhibitors of gastric acid secretion, such as proton-pump inhibitors (PPIs) [14]. However, NSAID-induced small intestinal damage has recently emerged as a clinically important issue [15]. To reduce the risk of complications, such as ulcers, bleeding, and obstruction, in the chronic NSAID users, many researches have attempted to treat and prevent these disorders [16]. Many drugs, such as metronidazole, sulfasalazine, COX2 inhibitors, misoprostol, and rebamipide, have been examined; however, these conditions remain incurable thus far [16]. DA9601, an ethanol extract of Artemisia asiatica, exhibit cytoprotective effect against various noxious agents through enhancement of prostaglandins and mucus secretion from gastric mucosa and is now used to treat acute and chronic gastritis in Korea $[10,17]$. It has also been reported that treatment with DA-9601 suppressed the expression of COX-2 and iNOS as well as binding of NF- $\kappa$ B to DNA in the colonic tissues. It also downregulated the phosphorylation of extracellular signal-regulated protein kinase and p38 mitogen-activated protein kinase, upstream of NF- $\mathrm{B}$ [18]. In this study, we attempted to induce enteritis following the administration of ACF. Inflammation of the small intestine was confirmed by histological studies, and the changes in the $\mathrm{CD}^{+} \mathrm{T}$ cell fraction according to inflammation-related cytokines and inflammatory reactions were then measured. In addition, by administering ESO or DA-9601 to experimental animal groups, we examined their possible therapeutic or preventive effects.

Through the preliminary evaluation, the optimal dose of ACF was chosen. In the group treated with ACF $(50 \mathrm{mg} / \mathrm{kg})$ for 2 days, apparent small intestine ulcers and inflammation were observed in most animals. Severe ulcers, necrosis, and extensive adhesions were observed in the high-dose groups $(100-200 \mathrm{mg} / \mathrm{kg}$ for 2 days). No apparent ulcers were observed in the group treated with $50 \mathrm{mg} / \mathrm{kg}$ ACF for one day. In addition to necrosis and adhesion of small intestinal lesions, exudative fluid was also observed in the abdominal cavity as an abscess in many gastrointestinal tracts; small intestinal lesions were observed in the lower part of the small intestine. These main lesions were observed as multifocal discrete small necrotic ulcers with intervening normal mucosa, which differed from the diffuse inflammatory lesions [19]. The data suggested that many lesions occurred due to the dissolution of the intestine by the administered ACF, as the mucous membrane between the small discrete ulcers was observed to be almost normal (i.e., inflammatory cell infiltration or villous blunting was rarely observed). In the histological analysis, the therapeutic effect of DA-9601 on ACF-induced enteritis was not observed. The lesions with multifocal discrete small necrotic ulcers were induced by ACF treatment; therefore, significant changes were 
not observed upon treatment with DA-9601. In addition, ESO treatment showed no protective effects on the lower small intestine, and even exacerbated symptoms, such as ulcer enlargement and necrosis, in the small intestine; these results corroborate with those previously reported $[20,21]$.

The transcription factor NF- $\kappa \mathrm{B}$ serves as a pivotal mediator of inflammatory responses. NF- $\kappa B$ induces the expression of various proinflammatory genes, including those encoding cytokines and chemokines, as well as participates in the inflammasome regulation. The development and progression of inflammation are mainly controlled by NF- $\mathrm{KB}$ activation. After activation, it can in turn activate the transcription of various genes and thereby regulate inflammation. Activation of NF- $\kappa \mathrm{B}$ increases the production of inflammatory cytokines, chemokines, and adhesion molecules at the site of inflammation [22]. The production of pro-inflammatory cytokines, such as IL-1, IL- 6 , IL-12, TNF- $\alpha$, and chemokines, leads to various inflammatory processes. It also promotes the differentiation of inflammatory $\mathrm{T}$ cells, including Th1 and Th17 cells, which in turn mediate the inflammatory responses [22]. Proinflammatory cytokines, such as IL-6, IL-17, and TNF- $\alpha$, have been reported to increase in Crohn's disease and ulcerative colitis [23]. TNF- $\alpha$ is known to act in T-helper 1 type (Th-1) disease, and IL-6, a proinflammatory cytokine, promotes IL-12 release and acts on T-helper 2 type (Th-2) cells in ulcerative colitis $[23,24]$. IL-17 is known to promote the inflammatory response by activating the $\mathrm{CD} 4^{+} \mathrm{T}$ cells by liberating IL-23 $[23,25]$.

Changes in the expression of genes encoding for IL-6, IL-17, and TNF- $\alpha$ were studied for the biochemical observation of inflammatory ulcers in the small intestine [24]. In this study, IL-6, $I L-17$, and TNF- $\alpha$ were found to have significantly increased in the ACF group, but their expression was significantly decreased in the ACF + DA-9601 and ACF + DA-9601 + ESO treated groups. However, the expression of these proinflammatory cytokines was significantly increased in the group treated with ACF + ESO as compared to that in the ACF treated group. These results suggest that treatment with ACF significantly increases the expression of proinflammatory cytokines, thereby enhancing the acute inflammation [26]. Administration of ESO increased the expression of proinflammatory cytokines, thereby increasing the acute inflammation as compared to that observed in the ACF treated group. However, treatment with DA-9601 might reduce the acute inflammatory reactions by inhibiting the expression of proinflammatory cytokines such as IL-6, IL-17, and TNF- $\alpha$ as observed in the ACF + DA-9601 and ACF + DA-9601 + ESO treated groups. These data suggest that DA-9601 prevents NSAID-induced small intestinal inflammation, which correlates with the reduction in proinflammatory cytokines.

In addition, the role of cytokines and chemokines, associated with inflammation, as inflammatory mediators was investigated. In the ACF treated group, IL- $1 \beta$, GM-CSF, IFN- $\gamma$, and TNF- $\alpha$ levels were significantly increased, whereas the IL-10 and IL-6 levels did not change. These data indicate that ACF induces an inflammatory reaction in the small intestine via changes in the inflammatory mediators. Treatment with DA-9601 significantly decreased the levels of proinflammatory mediators such as IL-1 $\beta$, GM-CSF, IFN- $\gamma$, and TNF- $\alpha$, but the anti-inflammatory cytokine IL-10 increased upon the treatment. These data suggest that the inflammatory response induced by ACF is reduced by DA9601 by controlling the production of cytokines and chemokines. In the ESO treated group, the changes in cytokines and chemokines were similar to those observed upon treatment with DA9601; however, the level of the anti-inflammatory cytokine IL-10 was found to have significantly decreased. These results showed that the administration of NSAIDs could increase inflammationrelated cytokines, in turn resulting in an inflammatory response in the small intestine. However, administration of DA-9601 could significantly decrease the proinflammatory cytokines and increase the anti-inflammatory cytokines, such as IL-10, which could inhibit the inflammatory response in the small intestine.

In inflammatory bowel disease, inflammatory mediators related to $\mathrm{T}$ cell imbalance and dysfunction are known to continuously activate the inflammatory response, causing chronic tissue damage $[27,28]$. In particular, it was reported that the levels of IL-17 and IL-23 were increased in the colonic mucosa of patients with Crohn's disease and ulcerative colitis and were related to the inflammatory response and disease severity $[29,30]$. Accordingly, we analyzed the phenotype of $\mathrm{CD} 4^{+} \mathrm{T}$ cells in ACF-induced enteritis to investigate the inflammatory mediators related to $\mathrm{T}$ cell imbalance and dysfunction. In this study, fractions of IFN- $\gamma^{+}$ Th1 cells, IL- $4^{+}$Th 2 cells, IL-17 $7^{+}$Th17 cells, and Foxp $3^{+}$Treg cells were significantly increased in the ACF treated group. However, the fractions of IL- $4^{+}$Th2 cells, IL-9 $9^{+}$Th9 cells, and Foxp3 ${ }^{+}$Treg cells were significantly decreased upon DA-9601 treatment. On the other hand, the fractions of IFN $-\gamma^{+}$Th1 cells, IL- $4^{+}$Th2 cells, IL- $9^{+}$Th9 cells, IL-17 $7^{+}$Th17 cells, and Foxp3 $3^{+}$Treg cells were increased by ESO treatment, suggesting that the acute inflammatory response mediated by T cells would be worsened by ESO treatment. These results are consistent with those obtained from studies on proinflammatory cytokine gene expression. The fractions of IFN- $\gamma^{+}$Th1 cells, IL- $4^{+}$Th2 cells, IL- $9^{+}$Th9 cells, IL-17 Th17 cells, and Foxp $3^{+}$Treg cells were decreased in the ACF + ESO + DA-9601 treated group and even recovered to the normal levels. These results suggest that in the case of co-administration of ESO and DA-9601, the acute inflammatory reaction mediated by $\mathrm{T}$ cells could be reversed to achieve normal (i.e., absence of inflammation) conditions.

Taken together, although additional studies with extensive control groups are needed to define the therapeutic effects of DA9601 on ACF-induced enteritis, our results strongly indicate that the treatment with DA-9601 could decrease the expression of cytokines and chemokines related to inflammation, as well as could inhibit the acute inflammatory response mediated by $\mathrm{T}$ cells in ACF-induced enteritis via suppression of NF- $\kappa \mathrm{B}$ activation and decreased COX-2 expression. 


\section{CONFLICTS OF INTEREST}

This study was sponsored by Dong-A ST Co., the manufacturer of DA-9601. Dong-A ST Co. made significant contributions to the design and analysis of the study. Except for CYS, SWJ, none of the investigators have a conflict of interest regarding this study.

\section{REFERENCES}

1. M'Koma AE. Inflammatory bowel disease: an expanding global health problem. Clin Med Insights Gastroenterol. 2013;6:33-47.

2. Podolsky DK. Inflammatory bowel disease. $N$ Engl J Med. 2002;347:417-429.

3. Jo-Watanabe A, Okuno T, Yokomizo T. The role of leukotrienes as potential therapeutic targets in allergic disorders. Int J Mol Sci. 2019;20:3580.

4. Ananthakrishnan AN, Higuchi LM, Huang ES, Khalili H, Richter JM, Fuchs CS, Chan AT. Aspirin, nonsteroidal anti-inflammatory drug use, and risk for Crohn disease and ulcerative colitis: a cohort study. Ann Intern Med. 2012;156:350-359.

5. Laine L, Smith R, Min K, Chen C, Dubois RW. Systematic review: the lower gastrointestinal adverse effects of non-steroidal antiinflammatory drugs. Aliment Pharmacol Ther. 2006;24:751-767.

6. Wallace JL, Syer S, Denou E, de Palma G, Vong L, McKnight W, Jury J, Bolla M, Bercik P, Collins SM, Verdu E, Ongini E. Proton pump inhibitors exacerbate NSAID-induced small intestinal injury by inducing dysbiosis. Gastroenterology. 2011;141:1314-1322, 1322. e1-e5.

7. Satoh H, Amagase K, Takeuchi K. Exacerbation of nonsteroidal anti-inflammatory drug-induced small intestinal lesions by antisecretory drugs in rats: the role of intestinal motility. J Pharmacol Exp Ther. 2012;343:270-277.

8. Lanas A, García-Rodríguez LA, Polo-Tomás M, Ponce M, AlonsoAbreu I, Perez-Aisa MA, Perez-Gisbert J, Bujanda L, Castro M, Muñoz M, Rodrigo L, Calvet X, Del-Pino D, Garcia S. Time trends and impact of upper and lower gastrointestinal bleeding and perforation in clinical practice. Am J Gastroenterol. 2009;104:1633-1641.

9. Legrand E. Aceclofenac in the management of inflammatory pain. Expert Opin Pharmacother. 2004;5:1347-1357.

10. Lee OY, Kang DH, Lee DH, Chung IK, Jang JY, Kim JI, Cho JW, Rew JS, Lee KM, Kim KO, Choi MG, Lee SW, Lee ST, Kim TO, Shin YW, Seol SY. A comparative study of DA-9601 and misoprostol for prevention of NSAID-associated gastroduodenal injury in patients undergoing chronic NSAID treatment. Arch Pharm Res. 2014;37:1308-1316.

11. Huh K, Kwon TH, Shin US, Kim WB, Ahn BO, Oh TY, Kim JA. Inhibitory effects of DA-9601 on ethanol-induced gastrohemorrhagic lesions and gastric xanthine oxidase activity in rats. J Ethnopharmacol. 2003;88:269-273.

12. Oh TY, Lee JS, Ahn BO, Cho H, Kim WB, Kim YB, Surh YJ, Cho SW, Lee KM, Hahm KB. Oxidative stress is more important than acid in the pathogenesis of reflux oesophagitis in rats. Gut. 2001;49:364-371.
13. Boynton CS, Dick CF, Mayor GH. NSAIDs: an overview. J Clin Pharmacol. 1988;28:512-517.

14. Scheiman JM. The use of proton pump inhibitors in treating and preventing NSAID-induced mucosal damage. Arthritis Res Ther. 2013;15(Suppl 3):S5.

15. Tai FWD, McAlindon ME. NSAIDs and the small bowel. Curr Opin Gastroenterol. 2018;34:175-182.

16. Park SC, Chun HJ, Kang CD, Sul D. Prevention and management of non-steroidal anti-inflammatory drugs-induced small intestinal injury. World J Gastroenterol. 2011;17:4647-4653.

17. Oh TY, Ryu BK, Ko JI, Ahn BO, Kim SH, Kim WB, Lee EB, Jin JH, Hahm KB. Protective effect of DA-9601, an extract of Artemisiae Herba, against naproxen-induced gastric damage in arthritic rats. Arch Pharm Res. 1997;20:414-419.

18. Kim HS, Kundu JK, Lee JS, Oh TY, Na HK, Surh YJ. Chemopreventive effects of the standardized extract (DA-9601) of Artemisia asiatica on azoxymethane-initiated and dextran sulfate sodiumpromoted mouse colon carcinogenesis. Nutr Cancer. 2008;60 Suppl 1:90-97.

19. Laine L. Nonsteroidal anti-inflammatory drug gastropathy. Gastrointest Endosc Clin N Am. 1996;6:489-504.

20. Blackler RW, Gemici B, Manko A, Wallace JL. NSAID-gastroenteropathy: new aspects of pathogenesis and prevention. Curr Opin Pharmacol. 2014;19:11-16.

21. Gwee KA, Goh V, Lima G, Setia S. Coprescribing proton-pump inhibitors with nonsteroidal anti-inflammatory drugs: risks versus benefits. J Pain Res. 2018;11:361-374.

22. Liu T, Zhang L, Joo D, Sun SC. NF- $\mathrm{B}$ signaling in inflammation. Signal Transduct Target Ther. 2017;2:17023.

23. Müzes G, Molnár B, Tulassay Z, Sipos F. Changes of the cytokine profile in inflammatory bowel diseases. World J Gastroenterol. 2012;18:5848-5861.

24. Strober W, Fuss IJ. Proinflammatory cytokines in the pathogenesis of inflammatory bowel diseases. Gastroenterology. 2011;140:17561767.

25. Kuwabara T, Ishikawa F, Kondo M, Kakiuchi T. The role of IL-17 and related cytokines in inflammatory autoimmune diseases. Mediators Inflamm. 2017;2017:3908061.

26. Chamoun-Emanuelli AM, Bryan LK, Cohen ND, Tetrault TL, Szule JA, Barhoumi R, Whitfield-Cargile CM. NSAIDs disrupt intestinal homeostasis by suppressing macroautophagy in intestinal epithelial cells. Sci Rep. 2019;9:14534.

27. Song EM, Jung SA, Lee JS, Kim SE, Shim KN, Jung HK, Yoo K, Park HY. Benzoxazole derivative B-98 ameliorates dextran sulfate sodium-induced acute murine colitis and the change of $\mathrm{T}$ cell profiles in acute murine colitis model. Korean J Gastroenterol. 2013;62:33-41.

28. Yamada A, Arakaki R, Saito M, Tsunematsu T, Kudo Y, Ishimaru N. Role of regulatory $\mathrm{T}$ cell in the pathogenesis of inflammatory bowel disease. World J Gastroenterol. 2016;22:2195-2205.

29. Cătană CS, Berindan Neagoe I, Cozma V, Magdaş C, Tăbăran F, Dumitraşcu DL. Contribution of the IL-17/IL-23 axis to the pathogenesis of inflammatory bowel disease. World J Gastroenterol. 2015;21:5823-5830.

30. Lee SH, Kwon JE, Cho ML. Immunological pathogenesis of inflammatory bowel disease. Intest Res. 2018;16:26-42. 